\title{
Dexmedetomidine and propofol for cerebral angiography in non-intubated patients: A comparative study
}

\author{
Sujoy Banik, Pallav Kumar1, Hemanshu Prabhakar, Gyaninder P. Singh
}

\begin{abstract}
Context: Patients posted for cerebral angiography may be restless and drowsy with high chance of inadvertent movements. Aims: The primary objective was to compare the incidence of inadvertent movements between propofol and dexmedetomidine groups. The secondary objectives include comparison of recovery time and characteristics, Steward's score, and haemodynamic and respiratory parameters between the two groups. Settings and Design: Prospective, randomised, double-blind, pilot study. Materials and Methods: In all, 20 adult uncooperative, drowsy patients were randomised to dexmedetomidine (I $\mu \mathrm{g} / \mathrm{kg}$ bolus over 10 minutes followed by $0.3-0.7 \mu \mathrm{g} / \mathrm{kg} / \mathrm{hour}$ infusion) or propofol $(100 \mu g / \mathrm{kg} / \mathrm{min}$ for 10 minutes followed by $25-75 \mu \mathrm{g} / \mathrm{kg} / \mathrm{min}$ infusion). Rate of movement, success of sedation, haemodynamics, respiratory parameters, Steward's recovery score and recovery time were recorded. Statistical analysis used: Repeated measures of analysis of variance, Mann-Whitney test, independent and paired t-tests, and Fisher test. Results: The median rate of movement was similar $(I, P=0.206)$ with success of sedation achieved in $7(70 \%)$ patients in Group $D$ and $9(90 \%)$ patients in Group $P$, which was comparable $(P=0.582)$. The median recovery time in patients in Group $D$ was 150 (37-764) seconds and in Group $P$ was $128(54-174)$ seconds $(P=0.519)$ with similar Steward's scores $(P=0.363)$. Haemodynamics and respiratory variables were well-maintained during loading and maintenance dose infusions in both the groups. Conclusions: Dexmedetomidine is a safe alternative for diagnostic cerebral angiography. Its success of sedation, median rate of movement during the imaging procedure, haemodynamics, respiratory parameters, recovery time and Steward's recovery score were similar to propofol in our study.
\end{abstract}

Key words: Cerebral angiography, dexmedetomidine, non-intubated, propofol, sedation

\section{INTRODUCTION}

Patients posted for cerebral angiography may be restless, drowsy and uncooperative, and pose a challenge to anaesthesiologists. Various anaesthetics such as propofol, midazolam and fentanyl have been tried earlier to sedate these patients during the procedure. ${ }^{[1]}$

\begin{tabular}{|l|l|}
\hline \multicolumn{2}{|c|}{ Access this article online } \\
\hline Quick Response Code: & Website: \\
\hline & www.jnaccjournal.org \\
\cline { 2 - 2 } & \\
\hline
\end{tabular}

Chances of inadvertent movements are high with these drugs especially during the femoral puncture and contrast injection. If the doses of sedatives are increased, then the incidence of airway obstruction is increased leading to high probability of airway manipulation with supra-glottic devices or endotracheal tube insertion.

Recently dexmedetomidine has been used for sedation in MRI suite ${ }^{[2,3]}$ and cardiac angiography. ${ }^{[4,5]}$ It is one of the most favoured drugs for conducting awake craniotomy. ${ }^{[6,7]}$ As both dexmedetomidine and propofol have short half-life and lead to early awakening after stopping the infusion, early post-anaesthetic care unit (PACU) discharge was anticipated. The primary objectives of our pilot study were to measure the rate of inadvertent movement and success of sedation during

Department of Neuroanaesthesiology, All India Institute of Medical Sciences, New Delhi, 'Department of Anaesthesia, Paras Hospital, Gurgaon, Haryana, India

Address for correspondence:

Dr. Sujoy Banik, GH-14 Flat No 747 PaschimVihar, Delhi - 110 087, India. E-mail: drsujoyb@gmail.com 
diagnostic cerebral angiography, in restless, drowsy and uncooperative patients. The secondary objectives of the study were to measure the recovery time after stoppage of sedation, using Steward's scoring system, ${ }^{[8]}$ and to compare the peri-procedural haemodynamic and respiratory parameters [heart rate (HR), oxygen saturation $\left(\mathrm{SpO}_{2}\right)$, non-invasive blood pressure (NIBP), end-tidal carbon dioxide $\left(\mathrm{EtCO}_{2}\right)$ and respiratory rate $(R R)]$.

\section{MATERIALS AND METHODS}

Twenty adult patients posted for diagnostic cerebral angiography in the neuroradiology suite were recruited for this prospective, randomised study. The study was approved by the ethics committee of the institute (IEC/NP-245/2012). Patients who were uncooperative, restless or drowsy, but responding to commands were included in the study. Patients not responding to verbal commands, age $<18$ years, and ASA grade IV and above were excluded from the study. Patients with anticipated difficult airway, on inotropic support, preoperative mechanical ventilation or sedative medication were also excluded from the study.

After obtaining written informed consent and pre-anaesthetic check-up, including routine investigations, the patients were shifted to the neuroradiology suite. Monitors were attached (five-lead ECG, NIBP, HR, RR monitor and pulse oximeter), and baseline parameters were recorded. Supplemental oxygen was provided by nasal prongs with integrated $\mathrm{EtCO}_{2}$ sampling line for monitoring $\mathrm{EtCO}_{2}$. Fentanyl $1 \mu \mathrm{g} / \mathrm{kg}$ was administered to all the patients. Both the patient and the assessor (neuroradiologist) were blinded to the study drug. Using a computer-generated randomisation chart and opaque-sealed envelopes, patients were allocated into two groups: Group D received dexmedetomidine $1 \mu \mathrm{g} / \mathrm{kg}$ bolus over 10 minutes followed by $0.3-0.7 \mu \mathrm{g} / \mathrm{kg} / \mathrm{hour}$ infusion; Group P received propofol $100 \mu \mathrm{g} / \mathrm{kg} / \mathrm{min}$ for 10 minutes followed by $25-75 \mu \mathrm{g} / \mathrm{kg} / \mathrm{min}$ infusion. Femoral puncture was allowed immediately after bolus was completed and local infiltration with lignocaine $2 \%$ was done. Time of starting study drug infusion and start of procedure were noted. Haemodynamic and respiratory parameters were recorded at 1-minute intervals during the period of bolus administration, and at every 5-minute intervals thereafter till the end of the procedure. Any movements during the procedure were noted and sedation adjusted accordingly. Rate of inadvertent movement during the imaging procedure was noted by the neuroradiologist, who was blinded to the study drug. A rescue bolus of propofol $20 \mathrm{mg}$ was administered if movement occurred despite maximal sedative infusion dose in both the groups. Successful sedation for the procedure was noted as the completion of diagnostic cerebral angiography without need for maintenance of airway using laryngeal mask airway (LMA) or endotracheal intubation.

If $\mathrm{SpO}_{2}$ continued to remain less than $90 \%$ for 30 seconds, then LMA was inserted to maintain adequate ventilation and oxygenation and the complication was noted. Bradycardia (HR decrease $>20 \%$ baseline) was treated with atropine $0.6 \mathrm{mg}$ and repeated if necessary. Tachycardia (HR increase $>20 \%$ baseline) was treated by fluid bolus and esmolol $0.5 \mathrm{mg} / \mathrm{kg}$ bolus if required. Hypotension (mean blood pressure decrease $<20 \%$ baseline) was treated by fluid bolus and mephentermine $3 \mathrm{mg}$ boluses, repeated every 3 minutes till normalisation of blood pressure, up to a maximum of $15 \mathrm{mg}$. Hypertension (blood pressure increase $>20 \%$ baseline) was treated by labetalol $10 \mathrm{mg}$ bolus repeated after 5 minutes if necessary. Number of such events was recorded along with the treatment given. Any other adverse effects like nausea, vomiting, retching, coughing, shivering and snoring were also recorded.

Study drug infusion was stopped immediately after dressing of the femoral puncture site. Patient was awakened, and time to response to commands noted. Post-procedural recovery score was recorded according to Steward's scoring system, ${ }^{[8]}$ which includes two points each for consciousness, airway (coughing on command or crying) and purposeful movement. One point is given for some response to stimuli but not being fully awake, maintaining good airway and non-purposeful movement, respectively; no points are awarded if the patient doesn't respond to commands, requires airway maintenance and/or no movement.

\section{Statistical analysis}

The descriptive data was expressed as mean with standard deviation, or median with range. The continuous variables were analysed by Kolmogorov-Smirnov test for normality. Repeated measures of analysis of variance were used to assess the change in continuous variables at multiple time points. Mann-Whitney test was used to analyse rate of movement and recovery time in both the groups. Fisher test was used to study success of sedation in the two groups. Paired t-tests were applied to the haemodynamic and respiratory variables, which were analysed separately during loading dose and maintenance dose infusion. Steward's recovery score was analysed by independent sample $t$-test in both the groups.

\section{RESULTS}

Twenty consecutive patients undergoing diagnostic cerebral angiography were enrolled in this pilot study, and data of all patients were analysed. None of the patients were excluded from final analysis. The 
demographic profile of the two groups shows that patients were well-matched for age, sex, and baseline haemodynamic and respiratory parameters [Table 1].

The median rate of movement noted was similar in both the groups $(1$, range $=0-3$ in both the groups, $P=0.206)$. Success of sedation was achieved in $7(70 \%)$ patients in Group D and 9 (90\%) patients in Group P, and were comparable $(P=0.582)$. The duration of procedure was $51 \pm 18$ minutes in patients in Group D, and $63 \pm 17$ minutes in patients in Group P, which was similar in both the groups $(P=0.210)$.

A decrease in HR and mean arterial pressure (MAP) was noted in patients of both Group D and Group P during loading dose infusion [Figure 1]. However the decrease in HR and MAP was comparable between both the groups $(P=0.64 ; P=0.48)$. Similarly there was a decrease in RR in patients in Group D and Group P $(P=0.002$ significant for Group P) during the loading dose infusion [Figure 2]. This decrease was comparable between the two groups $(P=0.699)$. During this time,

Table 1: Demographic and baseline characteristics of patients in study groups

\begin{tabular}{lccc}
\hline & Group D & Group P & $\boldsymbol{P}$ \\
\hline Age (years) & $45 \pm 13$ & $38 \pm 13$ & 0.221 \\
Weight (kg) & $64 \pm 15$ & $63 \pm 16$ & 0.791 \\
Sex (M/F) & $6 / 4$ & $7 / 3$ & 1.000 \\
Heart rate (/min) & $79 \pm 17$ & $73 \pm 11$ & 0.325 \\
Respiratory rate (/min) & $15 \pm 1$ & $16 \pm 4$ & 0.483 \\
EtCO 2 (mm Hg) & $32 \pm 8$ & $30 \pm 5$ & 0.423 \\
$\mathrm{MAP}(\mathrm{mm} \mathrm{Hg})$ & $105 \pm 22$ & $114 \pm 21$ & 0.571 \\
$\mathrm{SpO}_{2}(\%)$ & $99 \pm 1$ & $98 \pm 2$ & 0.732 \\
\hline
\end{tabular}

$\mathrm{EtCo}_{2}$ : End-tidal carbon dioxide, MAP: Mean arterial pressure, $\mathrm{SpO}_{2}$ : Oxygen saturation, Group D: Dexmedetomidine group, Group P: Propofol group

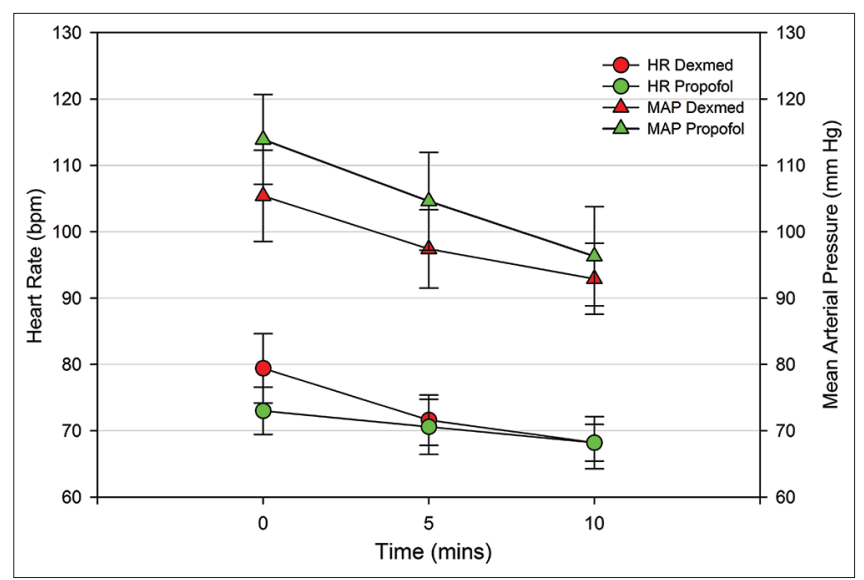

Figure 1: Graph showing changes in mean heart rate and mean arterial pressure during loading dose infusion of dexmedetomidine and propofol $\mathrm{HR}=$ Heart rate; $\mathrm{MAP}=$ Mean arterial pressure
$\mathrm{EtCO}_{2}$ showed an increase in patients from $31.9 \mathrm{~mm} \mathrm{Hg}$ to $35.8 \mathrm{~mm} \mathrm{Hg}$ in patients in Group D and from 29.5 $\mathrm{mm} \mathrm{Hg}$ to $30.4 \mathrm{~mm} \mathrm{Hg}$ in patients in Group $\mathrm{P}(P=0.048)$. This increase was higher in patients in Group $D$ than in Group P $(P=0.039)$ [Figure 2].

During maintenance dose infusion, insignificant fluctuation in mean $\mathrm{HR}, \mathrm{MAP}, \mathrm{RR}$ and $\mathrm{EtCO}_{2}$ were observed [Table 2]. Mean HR, MAP, RR and $\mathrm{EtCO}_{2}$ were similar throughout the maintenance dose infusion in both the groups [Figures 3 and 4]. The $\mathrm{SpO}_{2}$ was well-maintained in both groups at all measured time points (arterial puncture, sheath pullout, arousal) [Table 2].

The median recovery time in patients in Group D was 150 (37 - 764) seconds and in Group P was 128 (54-174) seconds $(P=0.519)$. The mean Steward's recovery score was $5.6 \pm 0.5$ in patients in Group D and $5.8 \pm 0.4$ in patients in Group P $(P=0.363)$.

Of the four patients who had failure of sedation, and were subsequently administered general anaesthesia, one patient in Group D did not allow arterial puncture, while 2 patients in Group D and 1 patient in Group $P$ had continuous movement hampering the imaging. The median total dose of dexmedetomidine used in Group D was $84 \mu \mathrm{g}$ (range $=58-137 \mu \mathrm{g}$ ), while the median total dose of propofol used in Group P was $200 \mathrm{mg}$ (range $=110-350 \mathrm{mg}$ ).

Rescue boluses were required in our study during the maintenance dose infusion once each in 2 patients in dexmedetomidine group (20 mg propofol each) and once in 1 patient in propofol group ( $20 \mathrm{mg}$ propofol). One patient required $1.2 \mathrm{mg}$ atropine to counteract bradycardia during maintenance dose infusion of dexmedetomidine. There were no other adverse effects noted intraoperatively or in immediate postoperative

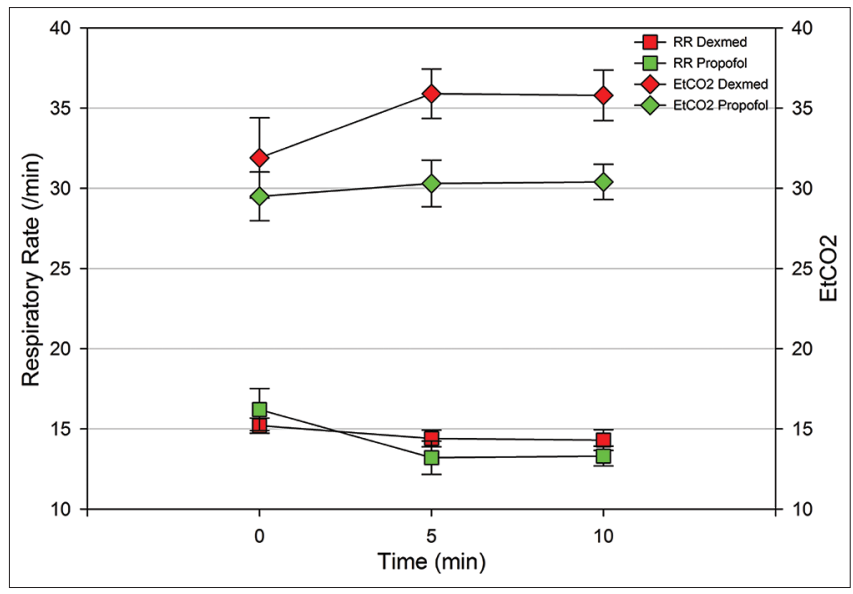

Figure 2: Graph showing changes in respiratory rate and $\mathrm{EtCO}_{2}$ during loading dose infusion of dexmedetomidine and propofol $\mathrm{RR}=$ Respiratory rate $; \mathrm{EtCO}_{2}=$ End-tidal carbon dioxide 
Table 2: Haemodynamic and respiratory parameters in patients in the two groups at various points of time (mean $\pm \mathrm{SD})$

\begin{tabular}{|c|c|c|c|c|c|c|c|c|c|}
\hline \multirow[t]{2}{*}{ parameters } & \multicolumn{3}{|c|}{ After arterial puncture } & \multicolumn{3}{|c|}{ After sheath pull out } & \multicolumn{3}{|c|}{ Arousal } \\
\hline & Group P & Group D & $P$ & Group P & Group D & $P$ & Group P & Group D & $P$ \\
\hline HR & $72 \pm 15$ & $69 \pm 14$ & 0.609 & $71 \pm 13$ & $75 \pm 19$ & 0.710 & $80 \pm 15$ & $79 \pm 18$ & 0.848 \\
\hline MAP & $95 \pm 11$ & $95 \pm 21$ & 0.968 & $83 \pm 18$ & $89 \pm 8$ & 0.441 & $91 \pm 20$ & $94 \pm 8$ & 0.688 \\
\hline $\mathrm{RR}$ & $14 \pm 3$ & $14 \pm 2$ & 0.586 & $15 \pm 2$ & $15 \pm 5$ & 0.954 & $15 \pm 2$ & $17 \pm 4$ & 0.362 \\
\hline $\mathrm{Spo}_{2}$ & $100 \pm 0$ & $99 \pm 1$ & 0.315 & $99 \pm 1$ & $100 \pm 0$ & 0.299 & $99 \pm 1$ & $100 \pm 0$ & 0.347 \\
\hline $\mathrm{Etco}_{2}$ & $35 \pm 7$ & $29 \pm 6$ & 0.058 & $36 \pm 6$ & $31 \pm 3$ & 0.083 & $34 \pm 4$ & $32 \pm 3$ & 0.324 \\
\hline
\end{tabular}

HR: Heart rate, MAP: Mean arterial pressure, RR: Respiratory rate, $\mathrm{SpO}_{2}$ : Oxygen saturation, EtCO : End-tidal carbon dioxide, Group D: Dexmedetomidine group, Group P: Propofol group

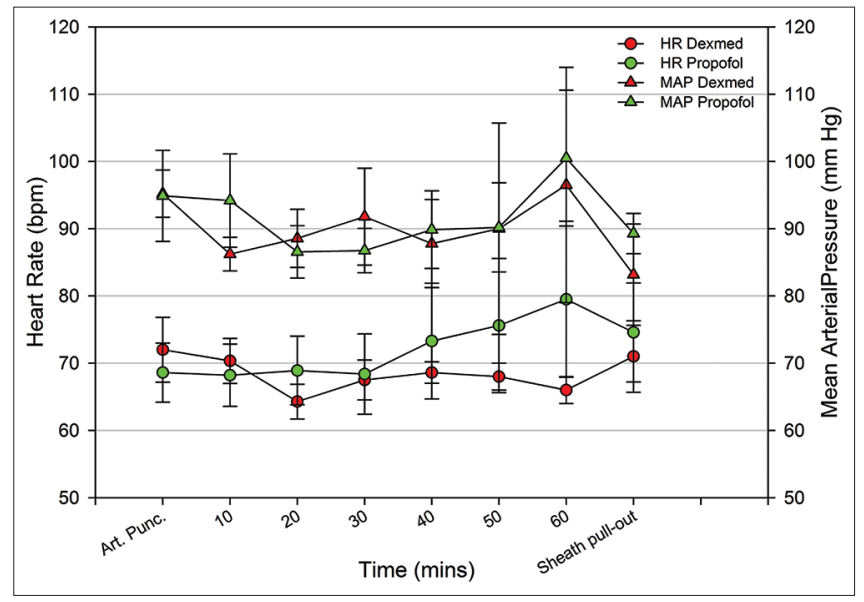

Figure 3: Graph showing changes in mean heartrate and mean arterial pressure during maintenance dose infusion of dexmedetomidine and propofol HR $=$ Heart rate; MAP = Mean arterial pressure. Art. Punc. $=$ Arterial puncture

period in either group. No vasopressors were used in either group. No respiratory depression requiring active intervention occurred in either of the two groups in our study.

\section{DISCUSSION}

Dexmedetomidine is a highly selective $\alpha_{2}$-agonist with dose-dependent sedative, anxiolytic and analgesic effects without ventilation suppression. In 1999, the American Food and Drug Administration approved the use of dexmedetomidine for sedation in ICU at a dose range of $0.2-0.7 \mu \mathrm{g} / \mathrm{kg} / \mathrm{hr}{ }^{\left[{ }^{6]}\right.}$ In 2001, Bekker et al. reported the first use of dexmedetomidine in awake craniotomy combined with LMA (spontaneous breathing), sevoflurane, fentanyl and nitrous oxide. ${ }^{[7]}$ The sedative, haemodynamic and respiratory effects of dexmedetomidine were evaluated and compared with those of midazolam in children undergoing MRI by Heard et al. ${ }^{[2]}$ Patients in dexmedetomidine group had a higher rate of imaging completion without the need to add another sedative (80\% compared with $20 \%$ in the midazolam group). In our study, success of sedation, as

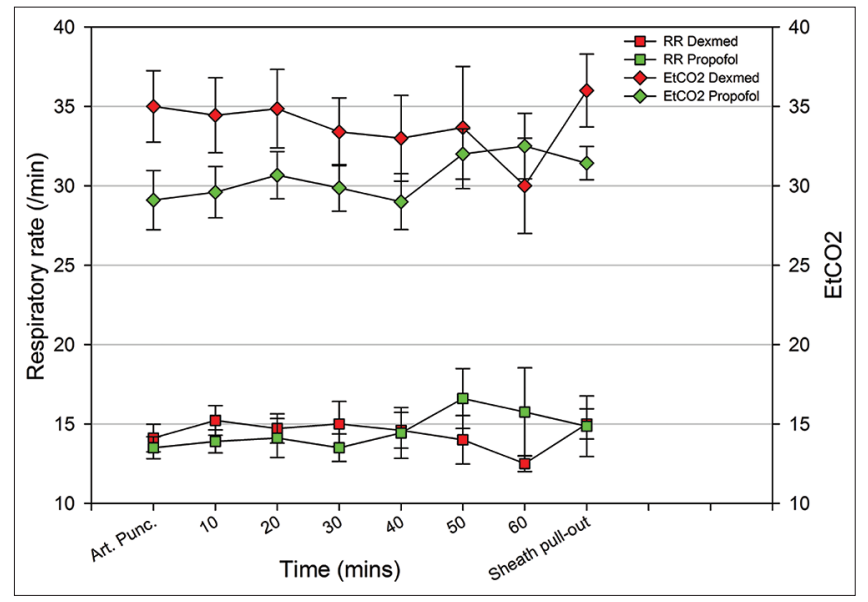

Figure 4: Graph showing changes in mean respiratory rate and end-tidal carbon dioxide during maintenance dose infusion of dexmedetomidine and propofolRR = Respiratory rate; $\mathrm{EtCO}_{2}=$ End-tidal carbon dioxide. Art. Punc. $=$ Arterial puncture

defined by completion of imaging procedure without supra-glottic device insertion or endotracheal intubation, was $70 \%$ with dexmedetomidine group, compared with $90 \%$ in propofol group. In 2006, Tosun et al. ${ }^{[4]}$ evaluated the effects of dexmedetomidine-ketamine and propofol-ketamine combinations on haemodynamics, sedation level, and the recovery period in paediatric patients undergoing cardiac catheterisation. The dexmedetomidine-ketamine combination was not better than propofol-ketamine combination because of a longer recovery time and inadequate sedation and analgesia. In a retrospective analysis by Mester et al., ${ }^{[5]}$ combination of ketamine and dexmedetomidine for sedation during cardiac catheterisation in children with congenital heart disease was found to provide effective sedation without significant effects on cardiovascular or ventilatory function. Wu et al. compared propofol and dexmedetomidine in children undergoing MRI. ${ }^{[3]}$ There were fewer pauses during MRI and lower failure rate of completion of MRI in propofol versus dexmedetomidine $(0.22$ versus $0.81, P=0.01$, and 1 versus $15, P<0.001)$, less behavioural disturbances 
in PACU, and better parental satisfaction in propofol versus dexmedetomidine $(P<0.01)$. They concluded that for children having long multi-component MRI, the propofol technique showed better outcomes than dexmedetomidine for PACU emergency, speed and parental satisfaction. Dexmedetomidine is a safe and useful drug for sedation during short surgical procedures. However, in our study, we found success of sedation was comparable using propofol and dexmedetomidine. The rate of movement was also similar in both the groups in our study.

Arain et al. ${ }^{[9]}$ studied the efficacy, side effects and recovery profile of dexmedetomidine infusion compared with propofol for intraoperative sedation in 40 patients for surgery under regional anaesthesia. They concluded that patients on dexmedetomidine had similar sedation to propofol, but with slower onset and offset of sedation and without respiratory depression. In our study, respiratory parameters and haemodynamics were well-maintained with dexmedetomidine and propofol throughout the procedure (during the loading dose as well as the maintenance dose) compared to Arain et al. where the MAP was lower during the intraoperative period, and this decrease was lesser in patients receiving dexmedetomidine $(11 \pm 3 \mathrm{~mm} \mathrm{Hg})$ (average intraoperative values, dexmedetomidine $86 \pm 3 \mathrm{~mm} \mathrm{Hg}$ versus propofol $75 \pm 3 \mathrm{~mm} \mathrm{Hg}$ ). This could be due to the effect of regional anaesthesia or the longer duration of the procedure in their study.

Propofol is commonly used for neurosurgical anaesthesia and awake craniotomy because of its easily titrable sedative effect and rapid recovery with clear-headedness. A combination of midazolam and opioids such as fentanyl may lead to increase in the respiratory depressive effects of either drug. Dexmedetomidine is quite unique in its ability to sedate without causing respiratory depression. It helps anaesthesiologists to facilitate a rapid patient wake up during neurosurgical procedures. ${ }^{[10]}$ Recently Shen et al. ${ }^{[11]}$ compared dexmedetomidine and propofol for awake craniotomy in 30 patients. They found significantly shorter arousal time with dexmedetomidine than propofol. Our results differ from those of Shen et al. with recovery time comparable but slightly longer with dexmedetomidine (150 seconds versus 128 seconds for propofol), yet rapid enough to allow for fast and reliable assessment of neurologic function post procedure. Our findings are not in accordance with those of Heard et al., ${ }^{[2]}$ whose results showed that the time to full recovery and to discharge from the ambulatory unit after dexmedetomidine administration were significantly longer (by $15 \mathrm{~min}$ ) than those after propofol. In the recovery room, dexmedetomidine was associated with an analgesia-sparing effect, slightly increased sedation, but no compromise of respiratory function or psychomotor performance. ${ }^{[9]}$ In our study, Steward's recovery score was comparable for both groups (5.6 \pm 0.5 for Group $\mathrm{D}$ and $5.8 \pm 0.4$ for Group P). There was no respiratory depression in either group in our study requiring active intervention.

Our study shows that dexmedetomidine has a similar rate of patient movement during neuroradiologic studies, while providing sedation similar to that obtained by use of propofol. However, our study is not without limitations. A larger sample size and the target-controlled infusion of propofol and dexmedetomidine could be used for more titrated sedation in a tightly controlled environment, as the slightest movement by the patient has an adverse impact on the quality of imaging by the neuroradiologist. The slight increase in $\mathrm{EtCO}_{2}$ levels seen in our study during maintenance dose infusion towards the end of the procedure could be attributed to errors inherent to the measurement of $\mathrm{EtCO}_{2}$ by nasal prongs, as it depended on both biological factors (tidal volume and RRs) and mechanical factors (the diameter and the length of the cannula and the diameter of the prongs ${ }^{[12]}$ Dexmedetomidine has been shown to prevent neurologic and cognitive testing in five patients during sedation for embolisation of cerebral arteriovenous malformation. ${ }^{[13]}$ However subarachnoid haemorrhage itself may impair cognition and consciousness, and may be a confounding factor for cognitive testing in this subset of patients in our study. Despite good outcome after subarachnoid haemorrhage, persistent cognitive consequences remain, limiting the patients' psychosocial functioning. ${ }^{[14]}$ Cognitive testing could have been performed after the study, despite the problems noted above and might be an important factor in overall outcome.

\section{CONCLUSION}

Dexmedetomidine is a safe and reliable alternative for sedation for diagnostic cerebral angiography in the neuroradiology suite without need for insertion of LMA or endotracheal intubation. Its success of sedation and median rate of movement during the imaging procedure were similar to propofol in our study. Haemodynamics and respiratory parameters were well-maintained in both the groups. Recovery time and Steward's recovery scores were similar in both the groups, demonstrating rapid and good recovery of patients from sedation for neurologic assessment.

\section{ACKNOWLEDGMENT}

Dr. Sujoy Banik: I would like to acknowledge the help of Dr. Suhas Singla, my good friend, for being a pillar of support at all times. 


\section{REFERENCES}

1. Bewlay MA, Laurence AS. Sedation for neuroradiology revisited: Comparison of three techniques for cerebral angiography. Eur J Anaesthesiol 2003;20:726-30.

2. Heard C, Burrows F, Johnson K, Joshi P, Houck J, Lerman J. A comparison of dexmedetomidine-propofol with midazolam for maintenance of anaesthesia in children undergoing magnetic resonance imaging. Anesth Analg 2008;107:1832-9.

3. Wu J, Mahmoud M, Schmitt M, Hossain M, Kurth D. Comparison of propofol and dexmedetomedine techniques in children undergoing magnetic resonance imaging. Paediatr Anaesth 2014;24:813-8.

4. Tosun Z, Akin A, Fuler G, Esmaoglu A, Boyaci A. Dexmedetomidine-ketamine and propofol-ketamine combinations for anesthesia in spontaneously breathing pediatric patients undergoing cardiac catheterization. J Cardiothorac Vasc Anesth 2006;20:515-9.

5. Mester R, Easley RB, Brady KM, Chilson K, Tobias JD. Monitored anesthesia care with a combination of ketamine and dexmedetomidine during cardiac catheterization. Am J Ther 2008;15:24-30.

6. Piccioni F, Fanzio M. Management of anaesthesia in awake craniotomy. Minerva Anestesiol 2008;74:393-408.

7. Bekker AY, Kaufmann B, Samir H, Doyle W. The use of dexmedetomidine infusion for awake craniotomy. Anesth Analg 2001;92:1251-3.

8. Steward DJ. A simplified scoring system for the post-operative recovery room. Canada Anaesth Soc J 1975;2:111-3.
9. Arain SR, Ebert TJ. The efficacy, side effects and recovery characteristics of dexmedetomidine versus propofol when used for intraoperative sedation. Anesth Analg 2002;95:461-6.

10. Shaukry M, Miller J. Update on dexmedetomidine: Use in nonintubated patients requiring sedation for surgical procedures. Ther Clin Risk Manag 2010;6:111-21.

11. Shen SL, Zheng JY, Zhang J, Wang WY, Jin T, Zhu J, et al. Comparison of dexmedetomidine and propofol for conscious sedation in awake craniotomy: A prospective, double-blind, randomized, and controlled clinical trial. Ann Pharmacother 2013;47:1391-9.

12. Fukuda $\mathrm{K}$, Ichinohe $\mathrm{T}$, Kaneko $\mathrm{Y}$. Is measurement of end-tidal CO2 through a nasal cannula reliable? Anesth Prog 1997;44:23-6.

13. Bustillo MA, Lazar RM, Finck AD, Fitzsimmons B, Berman MF, Pile-Spellman J, et al. Dexmedetomidine may impair cognitive testing during endovascular embolization of cerebral arteriovenous malformations: A retrospective case report series. J Neurosurg Anesthesiol 2002;14:209-12.

14. Ravnik J, Starovasnik B, Šešok S, Pirtošek Z, Švigelj V, Bunc G, et al. Long-term cognitive deficits in patients with good outcomes after aneurysmal subarachnoid hemorrhage from anterior communicating artery. Croat Med J 2006;47:253-63.

How to cite this article: Banik S, Kumar P, Prabhakar H, Singh GP. Dexmedetomidine and propofol for cerebral angiography in nonintubated patients: A comparative study. J Neuroanaesthesiol Crit Care 2015;2:121-6.

Source of Support: Nil, Conflict of Interest: None declared. 